Emir. J. Agric. Sci. 2006. 18 (2): 11-19

http://www.cfa.uaeu.ac.ae/research/ejas.htm

\title{
Influence of equilibration time during vitrification and culture medium on development of in vitro-derived bovine embryos
}

\author{
Abdulwali M. Al-aghbari ${ }^{1}$ and Alfred R. Menino, Jr. $^{2}$ \\ ${ }^{1}$ Department of Animal Science, Sana'a University, Sana'a, Yemen Republic \\ ${ }^{2}$ Department of Animal Sciences, Oregon State University, Corvallis, OR, U.S.A.
}

\begin{abstract}
The objective of the present study was to examine the influence of culture medium and equilibration time during vitrification on development of cryopreserved in vitro-derived bovine embryos. Following insemination, 50\% cumulus-enclosed presumptive zygotes were designated as controls and returned to the original microdrops for further incubation and the remaining of the embryos microdrops, randomly assigned into two culture groups, TCM-199 containing $10 \%(\mathrm{v} / \mathrm{v})$ fetal bovine serum and SOM prepared with the elevated potassium formulation (KSOM) supplemented with 1\%BSA were vitrified. Embryos were recovered from the cryo-vials and warmed at $37^{\circ} \mathrm{C}$ in $0.3 \mathrm{M}$ trehalose in TCM-199 for 1-2 min. Each group of embryos was immediately washed 3 times with its respective culture medium, KSOM or TCM, and returned to their corresponding microdrops containing the cumulus cell complement. Embryos were cultured for an additional seven days and evaluated at 24-h intervals to assess development. Development of presumptive bovine zygotes was similar in KSOM and TCM prior to vitrification. Development of bovine embryos was significantly greater $(\mathrm{P}<0.05)$ when equilibrated for $7 \mathrm{~min}$ than for 15 min although development of bovine embryos in TCM was similar regardless of the equilibration period. Development to the morula-blastocyst stage in KSOM for embryos equilibrated for 7 min exhibited similar $(\mathrm{P}>0.10)$ percentages to control and both were greater $(\mathrm{P}<0.05)$ than embryos equilibrated for 15 min or morula-blastocyst formation in TCM. This finding will enable more meaningful studies factors influencing development of embryos, which could allow establishment of embryo banks for the propagation of individuals with superior production traits or expansion of genetic diversity in populations with a waning genetic base.
\end{abstract}

Kay wards: Bovine, vitrification, Morula-blastocyst, embryos.

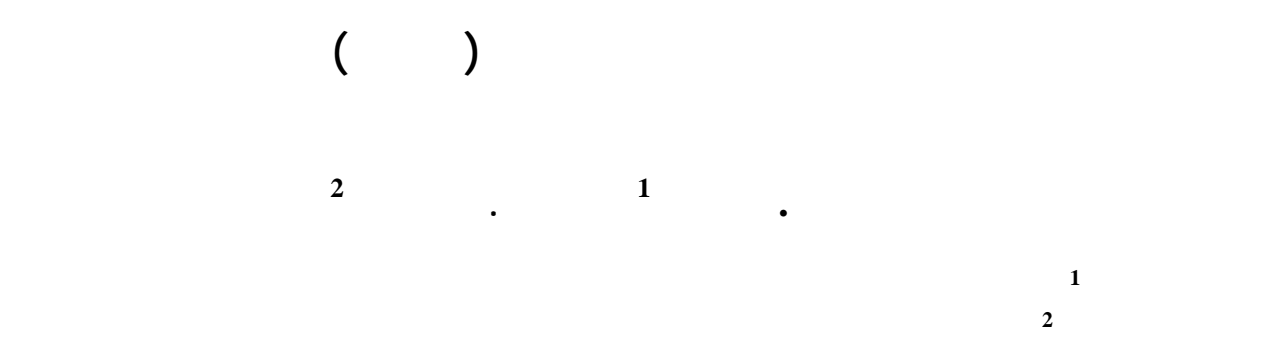

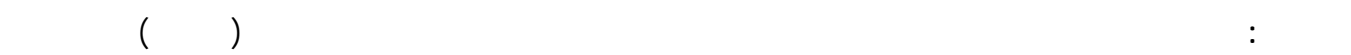

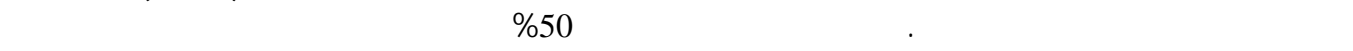

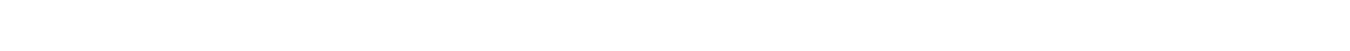

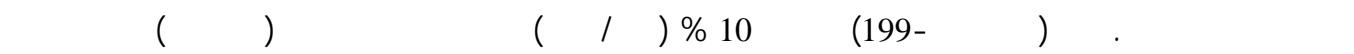

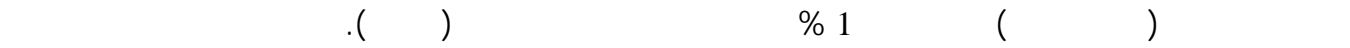

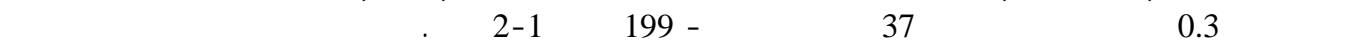

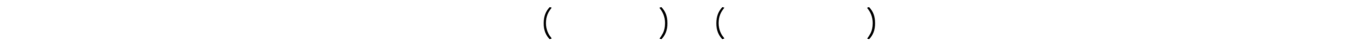

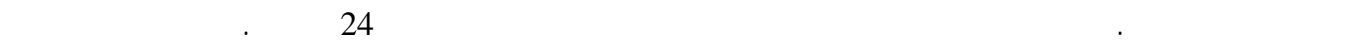

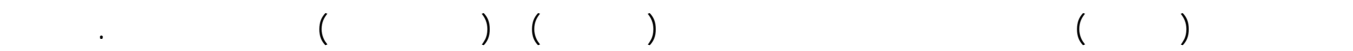
للأجنة القرية كلن معنويا (بي >

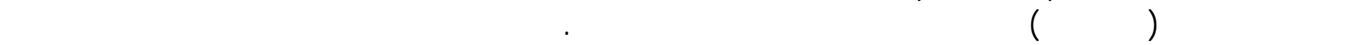

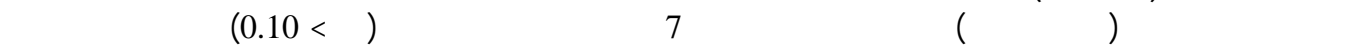

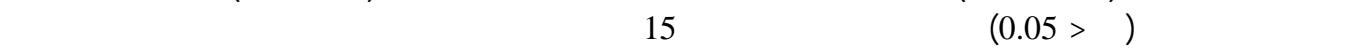


Abdulwali M. Al-aghbari et al.

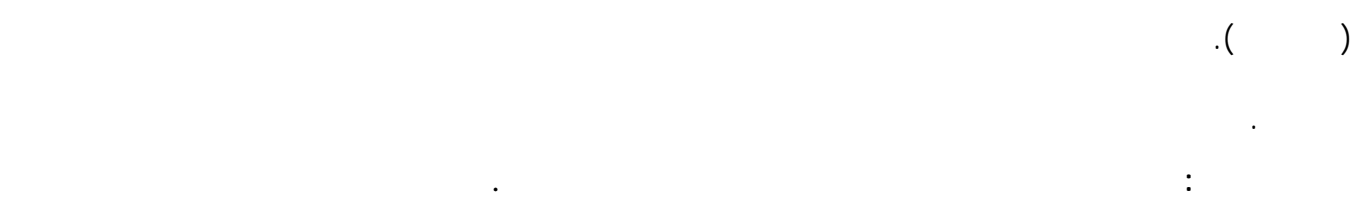

\section{Introduction}

Embryo transfer is an important tool for genetic improvement of livestock and conservation of endangered species. It allows establishment of embryo banks for the propagation of individuals with superior production traits or expansion of genetic diversity in populations with a waning genetic base (Gal et al., 2000; Ward et al., 2000, Al-aghbari, 2002; Nedambale et al., 2006). Embryos for such banks can be produced in vivo or by in vitro procedures.

In vitro embryo production enables the use of donors with high genetic merit that may be infertile or do not respond to conventional therapy such as superovulation (Ward et al., 2000; Rizos et al., 2001, Nedambale et al., 2006). In vitro embryo production for gene banking involves four steps: in vitro maturation of immature oocytes, in vitro fertilization of matured oocytes, in vitro culture of presumed zygotes and embryo cryopreservation. Oocytes are exposed to several processes through these stages, which ultimately affect the yield and quality of blastocysts (Greve et al., 1993; Carolan et al., 1995; Kaidi et al., 1998; Ward et al., 2000; Rizos et al., 2001, Kuwayama et al., 2005). In vitro-derived bovine blastocysts typically have reduced viability following cryopreservation compared to blastocysts obtained in vivo. Cryosurvival differences between in vivo and in vitro blastocysts have been reported by several laboratories (Pollard and Leibo, 1994; Kaidi et al., 1998; Crosier et al., 2000; Pugh et al., 2000; Fair et al., 2001; Chian et al., 2004). In vitro blastocysts have a greater amount of lipid, swollen blastomeres, darker cytoplasm and enhanced sensitivity to chilling. Attempts to improve survival rates of vitrified embryos have not been fully successful. Improvements in the vitrification technique could overcome the detrimental effect on viability induced by chilling injury (Kaidi et al., 1998; Dinnyes et al., 2000; Pugh et al., 2000). Numerous vitrification solutions (Saha and Suzuki, 1997) and types of freezers (Lim et al., 1991; Martino et al., 1996; Chian et al., 2004) have been used to reduce cryoprotectant toxicity, osmotic stress, and thermal injury, but major achievements in cryosurvival have been obtained by dramatically increasing the cooling rate (Dinnyes et al., 2000).

Major efforts have been directed at modifying post-maturation culture systems for in vitro-derived bovine embryos in order to improve overall development and tolerance to the extreme conditions of cryopreservation. Bovine embryos have been cultured in vivo in the ewe uterus or in vitro with somatic cells, such as oviductal or granulosa cells (Vajta et al., 1996; Martinez et al., 1998; Kaidi et al., 1998).

The beneficial effect of somatic cells on embryo development is still ambiguous. Basal media, used either alone or in combination with somatic cells, range from simple medium, like Synthetic Oviductal Medium (SOM) (Erbach et al., 1994; Enright et al., 2000), to more complex medium like TCM-199. Serum is frequently added to the culture medium and is purported to increase in vitro blastocyst viability by improving the morphological quality of morula (Crosier et al., 2000). 
Emir. J. Agric. Sci. 2006. 18 (2): 11-19

http://www.cfa.uaeu.ac.ae/research/ejas.htm

Because routine zygote culture in vivo is undesirable, it becomes essential to optimize in vitro culture systems. Therefore, the objective of the present study was to examine the influence of culture medium and equilibration time during vitrification on development of cryopreserved in vitro-derived bovine embryos.

\section{Materials and Methods}

Oocyte Collection and Maturation

Ovaries were collected from a regional slaughterhouse and transported to the laboratory (about $2 \mathrm{~h}$ ) in Dulbecco's PBS (DPBS). Upon arrival at the laboratory, ovaries were slashed with a scalpel and cumulus cell-oocyte complexes (COC) were recovered. Cumulus cell-oocyte complexes were washed three times in M2 medium and twice in maturation medium [TCM-199 (Sigma Chemical Co., St. Louis, MO) supplemented with $0.255 \mathrm{mM}$ sodium pyruvate (Sigma), 10\% heat-treated estrus cow serum and $10 \mathrm{ml} / \mathrm{l}$ antibioticantimycotic supplement (Sigma)] and cultured in 50- $\mu$ l microdrops of maturation medium under paraffin oil for 23-25 $\mathrm{h}$ at $39^{\circ} \mathrm{C}$ in a humidified atmosphere of $5 \%$ $\mathrm{CO}_{2}$ in air.

Sperm Preparation and In Vitro Fertilization (IVF)

Following maturation, COC were recovered from the culture drops and washed three times in Sperm-TALP (Parrish et al., 1986) before transfer into 50- $\mu$ l microdrops of fertilization medium (IVF-TALP; Parrish et al., 1986) supplemented with $0.4 \mathrm{mg} / \mathrm{ml}$ sodium heparin (Sigma). Motile spermatozoa were obtained from straws of frozen semen thawed by immersion in a $37^{\circ} \mathrm{C}$ water bath for 20 sec. Spermatozoa motility was enhanced by sperm "swim-up" method (Parrish et al., 1986) in 1-ml Sperm-TALP.
The upper fraction was recovered and centrifuged at $1000 \mathrm{X} \mathrm{g}$ for 6-7 min. The supernatant was discarded and spermatozoa were resuspended in $2 \mathrm{ml}$ of sperm-TALP and washed by centrifugation at $1000 \mathrm{X} \mathrm{g}$ for 6-7 min. Spermatozoa were counted in a hemocytometer and added to each $50-\mu \mathrm{l}$ microdrop of fertilization medium to a final concentration of $2 \times 10^{6}$ spermatozoa $/ \mathrm{ml}$. Cumulus cell-oocyte complexes were inseminated for $18 \mathrm{~h}$ at $39^{\circ} \mathrm{C}$ in a humidified atmosphere of $5 \% \mathrm{CO}_{2}$ in air.

\section{In Vitro Embryo Culture (IVC)}

Two types of IVC media were used in the study; TCM-199 containing 10\% (v/v) fetal bovine serum (FBS; Sigma), 0.255 $\mathrm{mM}$ sodium pyruvate and $10 \mathrm{ml} / \mathrm{l}$ antibiotic-antimycotic supplement (TCM) and SOM prepared with the elevated potassium formulation (KSOM) after Erbach et al. (1994), containing $10 \mathrm{ml} / \mathrm{l}$ antibiotic-antimycotic supplement and either $0.1 \%$ or $1 \%$ BSA (Sigma, catalog no. A4378). Following insemination, cumulusenclosed presumptive zygotes were randomly assigned into two culture groups, TCM or KSOM. The TCM group was washed three times with TCM and cultured in $50-\mu \mathrm{l}$ microdrops of this medium under paraffin oil in a humidified atmosphere of $5 \% \mathrm{CO}_{2}$ in air at $39^{\circ} \mathrm{C}$. Following $48 \mathrm{~h}$ of culture, embryos were denuded of surrounding cumulus cells by vortexing for 20-30 sec in $1 \mathrm{ml}$ of culture medium. Embryos were evaluated for cell stage and the number of two- to eight-cell embryos was recorded. Approximately 50\% of the embryos were designated as controls and returned to the original microdrops (12-15 embryos /microdrop) for further culture. The floors of the microdrops were partially covered by cumulus cells which had attached during culture of the zygotes. The remaining TCM embryos were vitrified. The KSOM group was treated similarly 
Abdulwali M. Al-aghbari et al.

except embryos were washed three times with $\mathrm{KSOM}+0.1 \%$ BSA and cultured in $50-\mu \mathrm{l}$ microdrops of this medium under paraffin oil. Following $48 \mathrm{~h}$ of culture, embryos were vortexed as described to remove the cumulus cells. Embryos were evaluated for cell stage and approximately $50 \%$ were returned to the original culture drops as controls. The remaining embryos were vitrified. In this system, the original medium had been carefully replaced; with $50 \mu \mathrm{l}$ of its respective culture (TCM or $\mathrm{KSOM}+1 \% \mathrm{BSA}$ ) so as not to disturb the adherent cumulus cells.

\section{Vitrification}

Embryos were vitrified according to the protocol described by Dinnyes et al. (2000). Briefly, embryos from both groups were washed three times in TCM-199 containing $0.255 \mathrm{mM}$ pyruvate, $25 \mathrm{mM}$ HEPES, $10 \mathrm{ml} / \mathrm{l}$ antibiotic-antimycotic supplement and $20 \%(\mathrm{v} / \mathrm{v})$ FBS and transferred into equilibration medium composed of $4 \%(\mathrm{v} / \mathrm{v})$ ethylene glycol (EG; Sigma) and 20\% (v/v) FBS in TCM-199 at $37{ }^{\circ} \mathrm{C}$ for either 7 or $15 \mathrm{~min}$. Embryos were transferred into vitrification solution containing $35 \% \quad(\mathrm{v} / \mathrm{v}) \quad \mathrm{EG}, 5 \% \quad(\mathrm{w} / \mathrm{v})$ polyvinylpyrrolidone (PVP, Sigma), $0.4 \mathrm{M}$ trehalose (Sigma) and 20\% (v/v) FBS in TCM-199 for 20-30 second. Embryos were dropped onto the surface of a steel cube, covered with aluminum foil and cooled by liquid nitrogen as described by Dinnyes et al. (2000). Vitrified embryos were placed in $1 \mathrm{ml}$ cryo-vials for $24 \mathrm{~h}$ in liquid nitrogen.

\section{Warming}

Embryos were recovered from the cryovials and warmed at $37^{\circ} \mathrm{C}$ in $0.3 \mathrm{M}$ trehalose in TCM-199 for 1-2 min. Each group of embryos was immediately washed 3 times with its respective culture medium, $\mathrm{KSOM}+1 \% \mathrm{BSA}$ or TCM, and returned to their corresponding microdrops containing the cumulus cell complement. Embryos were cultured for an additional seven days and evaluated at 24-h intervals to assess development.

\section{Statistical analysis}

Zygotes were assigned an embryo development score based on the terminal cell stage achieved in culture: $1=2$-cell, 2=4-cell, 3=8-cell, 4=16-cell, 5=morula and 6=blastocyst. Embryo development scores for zygotes cultured during the 48-h interval prior to vitrification were analyzed for differences due to medium using the pooled t-test. Differences in embryo development scores following vitrification were analyzed using a $2 \times 3$ factorial design where the main effects were Medium, KSOM or TCM, and Treatment, control (not vitrified) and 7 or $15 \mathrm{~min}$ equilibration in cryoprotectant prior to freezing. Differences between main effects means or individual means were analyzed using the Duncan's multiple comparison test. Differences due to treatment in the percentages of embryo developing into morulae and blastocysts were analyzed using Chi-square procedures for contingency tables. All analyses were performed using the NCSS statistical software program.

\section{Results}

Embryo development scores were similar for zygotes developing in KSOM $(1.80 \pm 0.06 ; \mathrm{n}=181)$ and TCM $(1.81 \pm 0.06$; $\mathrm{n}=195)$ prior to vitrification. Culture medium did not affect $(\mathrm{P}>0.10)$ embryo development score following vitrification and the Medium X Treatment interaction was not significant (Table 1). Treatment greatly affected embryo development where control embryos had greater $(\mathrm{P}<0.05)$ embryo development scores than embryos incubated in equilibration solution 
Emir. J. Agric. Sci. 2006.18 (2): 11-19

http://www.cfa.uaeu.ac.ae/research/ejas.htm

for 7 or $15 \mathrm{~min}$ prior to vitrification and culture (Table 1). Also, embryos equilibrated for $7 \mathrm{~min}$ had greater $(\mathrm{P}<0.05)$ embryo development scores than embryos equilibrated for 15 min (Table 1). Analysis of the individual means revealed specific differences in embryo performance due to a particular combination of equilibration time and culture medium. For example, embryos equilibrated for $15 \mathrm{~min}$, vitrified and cultured in KSOM had lower $(\mathrm{P}<0.05)$ embryo development scores than embryos equilibrated for $7 \mathrm{~min}$ or control embryos, regardless of the culture medium (Figure 1). However, embryos equilibrated for 15 min, vitirified and cultured in TCM had embryo development scores similar $(\mathrm{P}>0.10)$ to any group of embryos cultured in KSOM and embryos equilibrated for 7 min and cultured in TCM (Figure 1). Only control embryos cultured in TCM had greater $(\mathrm{P}<0.05)$ development scores than embryos equilibrated for $15 \mathrm{~min}$, vitrified and cultured in TCM (Figure 1).

Analysis of the percentages of embryos attaining the morula-blastocyst stage demonstrated differences in development that were not revealed by analysis of the embryo development scores. Development to the morula-blastocyst stage in KSOM followed the identical trend observed for embryo development score where control embryos and embryos equilibrated for 7 min exhibited similar $(\mathrm{P}>0.10)$ percentages and both were greater $(\mathrm{P}<0.05)$ than embryos equilibrated for 15 min (Figure 2). Morula-blastocyst formation in TCM deviated from embryo development score because similar percentages of embryos equilibrated for 7 and 15 min developed to this stage but both were lower $(\mathrm{P}<0.05)$ compared to control embryos (Figure 2). When comparisons were conducted within an equilibration time, no differences $(\mathrm{P}>0.10)$ in percent morula-blastocysts were observed due to culture medium.

Table 1. Main effects means of embryo development scores for zygotes equilibrated in cryoprotectant for 7 or 15 min prior to vitrification and cultured in KSOM or TCM after warming.

\begin{tabular}{lccc}
\hline Main effects & n & Mean & SE \\
\hline Medium & & & \\
KSOM & 181 & $2.55^{\mathrm{a}}$ & 0.12 \\
TCM & 195 & $2.74^{\mathrm{a}}$ & 0.11 \\
& & & \\
Treatment & 161 & $3.07^{\mathrm{a}}$ & 0.12 \\
$\quad$ Control (not vitrified) & 112 & $2.63^{\mathrm{b}}$ & 0.15 \\
7-min equilibration & 103 & $2.02^{\mathrm{c}}$ & 0.15 \\
15-min equilibration &
\end{tabular}

${ }_{\text {a,b,c }}$ Means within a main effect without common superscripts are different $(P<0.05)$. 
Abdulwali M. Al-aghbari et al.

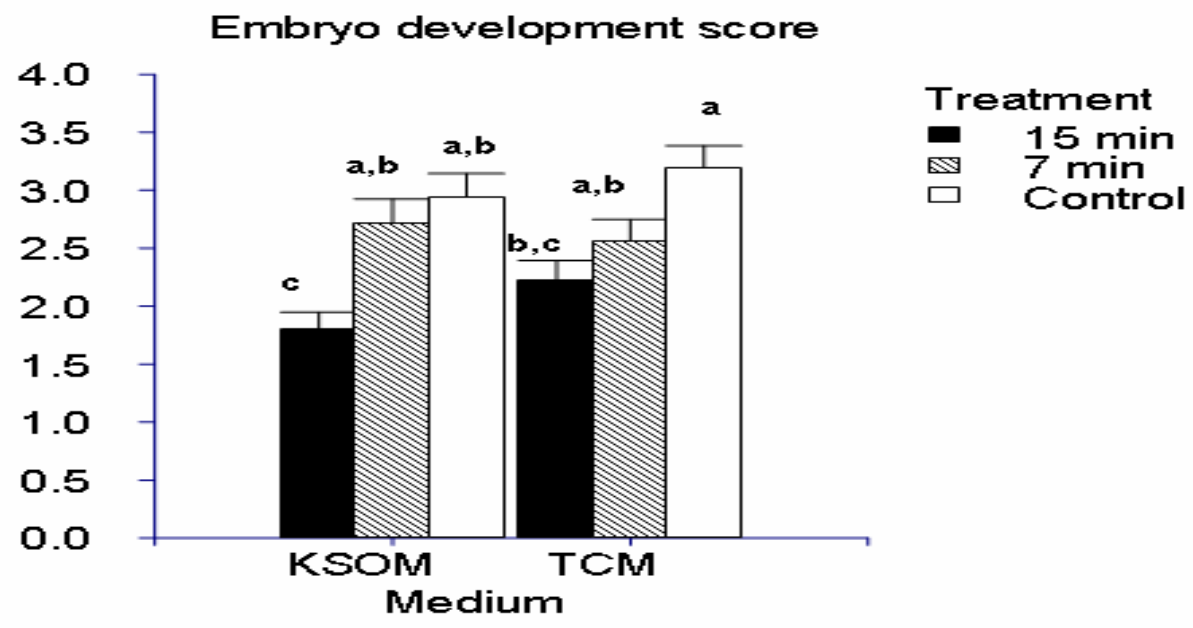

a,b,c Means without common superscripts differ $(\mathrm{P}<0.05)$

Figure 1. Embryo development scores of zygotes equilibrated in cryoprotectant for 7 or 15 min prior to vitrification and cultured in KSOM or TCM after warming.

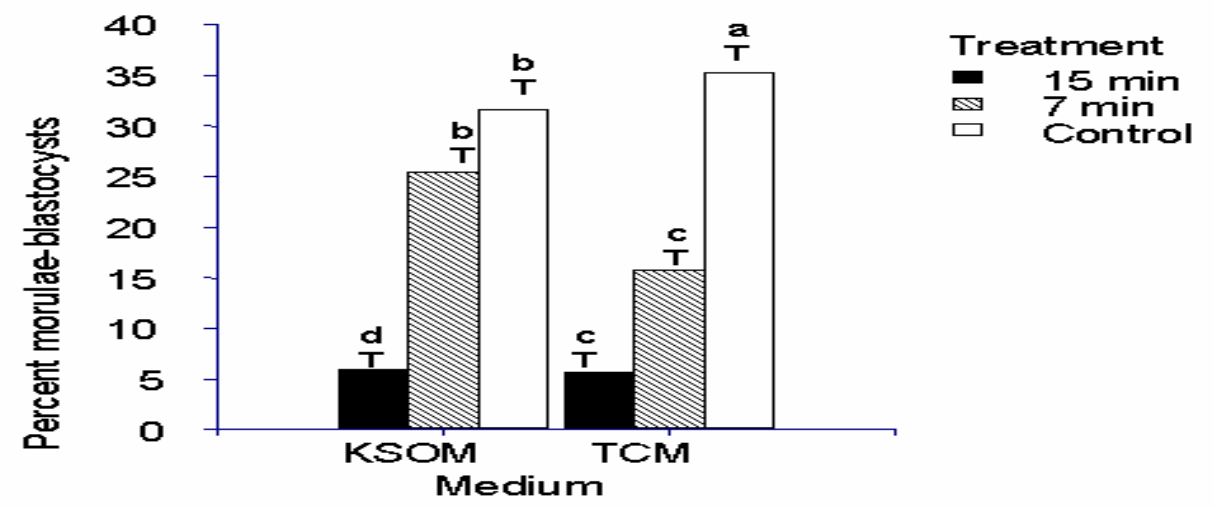

a, b, c, d Percents for a specific culture medium without common superscripts differ $(\mathrm{P}<0.05)$.

Figure 2. The percentages of embryos attaining the morula-blastocyst stage that were equilibrated in cryoprotectant for 7 or $15 \mathrm{~min}$ prior to vitrification and cultured in KSOM or TCM after warming.

\section{Discussion}

Culture of bovine zygote in vivo attendance to be undesirable for both practical and animal welfare, (Vajta et al., 1996; Martinez et al., 1998; Kaidi et al., 1998; Rizos et al., 2001; Pugh et al., 2000, Kuwayama et al., 2005) thus, it becomes essential to optimize in vitro culture systems. The present study was designed to investigate the developmental differences of bovine embryos in KSOM and TCM culture medium and the influence of equilibration time during vitrification on morula-blastocyst development rates. The most important findings from this study were as follow: (1) development of presumptive bovine zygotes is similar in KSOM and TCM prior to vitrification. (2) Development of bovine embryos is greater when equilibrated for 7 min than for 15 min although development of bovine 
Emir. J. Agric. Sci. 2006. 18 (2): 11-19

http://www.cfa.uaeu.ac.ae/research/ejas.htm

embryos in TCM were similar regardless of the equilibration period. (3) When embryo equilibrated for $7 \mathrm{~min}$ and cultured in KSOM after vitrification/warming, embryo development to morula-blastocyst stage was similar to the control group. Our results in Table 1 demonstrated that the development of presumptive bovine zygotes is similar in KSOM and TCM prior to vitrification. While these results support the major efforts that have been directed at modifying maturation and fertilization culture systems for in vitro-derived bovine embryos (Erbach et al., 1994; Enright et al., 2000, Laowtammathron et al., 2005), it would suggest that the process of maturation and fertilization might not be the main factors influencing the morulablastocyst yield and quality. Therefore, equilibration time with cryoprotectant agents, protocol of vitrification and post vitrification culture might have the most impact on both post thaw embryo development and the yield of morulablastocyst.

High rates of bovine embryo viability were observed following vitrification as shown in Table 1 and Figure 1. In agreement with previous report (AlAghbari and Menino 2002) dropping embryo equilibrated for 7 or $15 \mathrm{~min}$ directly onto a solid surface cooled by liquid nitrogen has cryosurvival rates superior compared to methods using conventional freezing techniques. We demonstrated that while embryo viability equilibrated for $7 \mathrm{~min}$ was high in KSOM and TCM and was similar to embryo equilibrated for $15 \mathrm{~min}$ in TCM, the quality and yields of morula-blastocysts were dependable on the type of the postwarming embryo culture. As shown in Figure 2 the differences in the yields of morula-blastocysts between the postwarming embryo cultures were significantly high. Post-warming embryo equilibrated for $7 \mathrm{~min}$ and culture in
KSOM yields morula-blastocyst similar to non-vitrified control. In contrast, the development of morula-blastocyst in TCM was significantly low and was deviated from the viability of post-warming embryo as shown in Figure 1. Post-warming embryo viability can be evaluated by different methods the most common is in vitro survival following a period of culture. Several culture systems have been designed for this purpose (Kaidi et al., 1998; Rizos et al., 2001; Pugh et al., 2000, Kuwayama $\mathrm{M}$ et al., 2005, Nedambale et al., 2006). When Rizos et al. (2001) compared post-warming viability of vitrified bovine embryos on SOF and TCM, it found that blastocysts yields were higher in SOF than TCM. These researchers indicated that development of post-warming embryo to blastocysts is dramatically affected by the culture system used. In contrast, Saha and Suzuki (1997) and Pugh et al. (2000) indicated that addition of trehalose and PVP to $20-40 \%$ EG as cryprotectant solution allowed more embryos to survive to hatching. Therefore, several factors might have led to the high morula-blastocysts yields in the present study: 1) addition of trehalose and PVP to 20-40\% Eg to cryprotectant solution, 2) reducing equilibration time from 15 to 7 min., 3) dropping equilibrated embryos directly onto a solid surface cooled by liquid nitrogen, and 4) using $\mathrm{KSOM}$ as the culture system.

In conclusion, we demonstrated that development of post-warming embryos to morula-blastocysts is not dependable on percentage of embryo viability but on the type of the culture system used. Using $\mathrm{KSOM}$ as the culture system and reducing equilibration time from 15 to $7 \mathrm{~min}$ undoubtedly improves the yields of morula-blastocysts. We also confirmed the benefits of adding trehalose and PVP to Eg as cryprotectant solution, and dropping equilibrated embryos directly onto a solid 
surface cooled by liquid nitrogen on the quality of embryos produced. This finding will enable more meaningful studies factors influencing development of embryos, which could allow establishment of embryo banks for the propagation of individuals with superior production traits or expansion of genetic diversity in populations with a waning genetic base.

\section{Acknowledgments}

The authors wish to express sincere appreciation to Islamic Development Bank and Oregon State University for their support of this investing.

\section{Referentes}

Al-aghbari A. M. 2002. The effect of vitrification on survival of bovine oocytes recovered from ovarian tissues. Faculty of Sci. Bulletin 15:123-132.

Al-aghbari A. M. and A. R. Menino, Jr 2002. Survival of oocytes recovered from vitrified sheep ovarian tissues. Anim. Reprod. Sci. 71:101-110.

Carolan, C., P. Lonergan, A., Van Langendonckt and P. Mermillod. 1995. Factors affecting bovine embryo development in synthetic oviduct fluid following oocyte maturation and fertilization in vitro. Theriogenology 43:15-1128.

Chian R. C, M. Kuwayama Tan L, J. Tan, O. Kato, T. Nagai. 2004. High survival rate of bovine oocytes matured in vitro following vitrify-cation. J. Reprod Dev. 50(6):685-696.

Crosier, A. E., P. W. Farin, M. J. Dykstra, J. E. Alexander and C. E. Farin. 2000. Ultrastructural morpho-metry of bovine compact morulae produced in vivo or in vitro. Biol. Reprod. 62:1459-1465.

Dinnyes, A., Y. Dai, S. Jiang and X. Yang, 2000. High developmental rates of vitrified bovine oocytes following parthenogenetic acti-vation, in vitro fertilization, somatic cell nuclear transfer. Biol. Reprod. 63:513-518.

Enright, B. P., P. Lonergan, A. Dinnyes, T. Fair, F. A. Ward, X. Yang, M. P. Boland. 2000. Culture of in vitro produced bovine zygotes in vitro vs in vivo: implications for early embryo development and quality. Theriogenology 54:659-673.

Erbach, G. T., J. A. Lawitts, V. E. and J. D.Papaioannou. 1994. Diffe-rential growth of the mouse preimplantation embryo in chemically defined media. Biol. Reprod. 50: 1027-1033.

Fair, T., P. Lonergan, A. Dinnyes, D. C. Cottell, P. Hyttel, F. A. Ward and M. P. Boland, 2001. Ultrastructure of bovine blastocysts following cryopreservation: Effect of method of blastocysts production. Mol. Reprod. Dev. 58: 186-195.

Gal, F., R. Roover, B. Verhaeghe, D. Etienne and A. Massip. 2000. Development of vitrified matured cattle oocytes after thawing and culture in vitro. Vet. Record. 146: 469-471.

Greve, T., B. Avery and H. Callensen 1993. Viability of in vivo and in vitro produced bovine embryos. Reprod. Domest. Anim. 28: 164-169.

Kaidi, S., I. Donnay, A. Van Langendonckt, F. Dessy and A. Massip. 1998. Comparison of two coculture systems to assess the survival of 
Emir. J. Agric. Sci. 2006. 18 (2): 11-19

http://www.cfa.uaeu.ac.ae/research/ejas.htm

in vitro produced bovine blastocysts after vitrification. Anim. Reprod. Sci. 52: 39-50.

Kuwayama M, G. Vajta, O. Kato, P. Leibo. 2005. Highly efficient vitrification method for cryopreservation of human oocytes. Reprod Biomed Online. 11 (3):300-308.

Laowtammathron, C., C. Lorthongpanich, M. Ketudat-Cairns, S. Hochi, R. Parnpai. 2005. Factors affecting cryosurvival of nuclear-transferred bovine and swamp buffalo blastocysts: Effects of hatching stage, linoleic acidalbumin in IVC medium and Ficoll supplementation to vitrification solution. Theriogenology. 64 (5):11851196.

Lim, J. M., Y. Fukui and H. Ono. 1991. The post-thaw developmental capacity of frozen bovine oocytes following in vitro maturation and fertilization. Theriogenology 35: 1225-1235.

Martino, A., N. Songansen and S. P. Leibo 1996. Development into blastocysts of bovine oocytes crypreserved by ultrarapid cooling of very small samples. Biol. Reprod. 54:1059-1069.

Martinez, A. G., D. G. De Matos, C. C. Furnus and G. M. Brogliatti. 1998. In vitro evaluation and pregnancy rates after vitrification of in vitro produced bovine embryos. Therio-genology 50:757-767.

Nedambale, T. L, F. Du, X. Yang, X. C. Tian. 2006. Higher survival rate of vitrified and thawed in vitro produced bovine blastocysts following culture in defined medium supplemented with beta-mercapto-ethanol. Anim Reprod Sci 93(1-2):61-75.

Parrish, J. J., J. Susko-Parrish, M. L. Leibfried-Rutledge, E. S. Critser, W. H. Eyestone. 1986. First NL. Bovine in vitro fertilization with frozen-thawed semen. Theriogenology 25, 591-600.

Pollard, J. W., S. P. Leibo. 1994. Chilling sensitivity of mammalian embryos. Theriogenology 41, 101-106.

Pugh, P. A., H. R. Tervit, H. Niemann. 2000. Effect of vitrification medium composition on the survival of bovine in vitro produced embryos, following in straw-dilution, in vitro and in vivo following transfer. Anim. Reprod. Sci. 58, 9-22.

Rizos, D., F. A. Ward, M. P. Boland, P. Lonergan. 2001. Effect of culture system on the yield and quality of bovine blastocysts as assessed by survival after vitrification. Theriogenology 56, 1-16.

Saha, S., T. Suzuki. 1997. Vitrification of in vitro produced bovine embryos at different ages using one- and three-step addition of cryoprotective additives. Reprod. Fertil. Dev. 7, 741-746.

Vajta, G., P. Holm, T. Greve, H. Callensen. 1996. Overall efficiency of in vitro embryo production and vitrification in cattle. Theriogenology 45, 683-689.

Ward, F. A., P. Lonergan, B. P. Enright, M. P. Boland. 2000. Factors affecting recovery and quality of oocytes for bovine embryo production in vitro using ovum pick-up technology. Theriogenology 54, 433-446. 